\title{
Thermodynamic geometry and phase transition of spinning AdS black holes
}

\author{
Amin Dehyadegari* and Ahmad Sheykhi ${ }^{\dagger}$ \\ Department of Physics, College of Sciences, Shiraz University, Shiraz 71454, Iran \\ and Biruni Observatory, College of Sciences, Shiraz University, Shiraz 71454, Iran
}

(Received 7 September 2021; accepted 8 November 2021; published 30 November 2021)

\begin{abstract}
Employing the thermodynamic geometry approach, we explore the phase transition of four-dimensional spinning black holes in an anti-de Sitter (AdS) space and find the following novel results. (i) Contrary to the charged AdS black hole, thermodynamic curvature of the spinning AdS black hole diverges at the critical point, without needing normalization. (ii) There is a certain region with small entropy in the space of parameters for which the thermodynamic curvature is positive and the repulsive interaction dominates. Such behavior exists even when the pressure is extremely large. (iii) The dominant interactions in the microstructure of extremal spinning AdS black holes are strongly repulsive, which is similar to an ideal gas of fermions at zero temperature. (iv) The maximum of thermodynamic curvature, $|R|$, is equal to $C_{P}$ maximum values for the Van der Waals fluid in the supercritical region, while for the black hole, they are close to each other near the critical point.
\end{abstract}

DOI: 10.1103/PhysRevD.104.104066

\section{INTRODUCTION}

Thermodynamic fluctuation provides a unique framework for the geometrical description of thermodynamical systems in equilibrium. We are particularly interested in the covariant version, known as Ruppeiner geometry [1], which consists of a metric that measures the probability of a fluctuation between two thermodynamic equilibrium states. The Riemannian scalar curvature, known as the thermodynamic curvature, arises from such a metric and is a fundamental object in the Ruppeiner geometry which contains information about interparticle interaction. More specifically, a negative (positive) sign of the thermodynamic curvature determines an attractive (repulsive) interaction between particles, while a zero value for the thermodynamic curvature means there is no interaction between particles [2-4]. The absolute value of the thermodynamic curvature in the asymptotic critical region is related to the correlation length in fluids [3].

Since the discovery of entropy and temperature of black holes $[5,6]$, it has been well established that one can regard a black hole as a thermodynamic system characterized by a set of thermodynamic variables. It is particularly interesting to investigate the critical behavior and phase transition of

\footnotetext{
*amindehyadegari@gmail.com

asheykhi@shirazu.ac.ir
}

Published by the American Physical Society under the terms of the Creative Commons Attribution 4.0 International license. Further distribution of this work must maintain attribution to the author(s) and the published article's title, journal citation, and DOI. Funded by SCOAP. black holes in the background of AdS spacetime [7-11]. It has been confirmed that one can extend the thermodynamic phase space by treating the cosmological constant as the thermodynamic pressure, $P=-\Lambda /(8 \pi)$, in an extended phase space, with its conjugate variable as volume [12-17]. In this regard, continuous and discontinuous phase transitions between small and large charged AdS black holes have been realized [18], which are analogous to the Van der Waals liquid-gas phase transition and belong to the same universality class $[18,19]$. Besides, these studies reveal some interesting phenomena, such as the Van der Waals liquid-vapor phase transition [18], the zeroth-order phase transition [20], the reentrant phase transition [21,22], the triple critical point [23], the superfluid-like phase transition [24], and many others.

In the context of black hole thermodynamics, the thermodynamic curvature in the Ruppeiner geometry provides a powerful tool to explore the microscopic behavior of black holes. The results obtained can also be compared with accessible experimental systems. Thermodynamic curvature has been investigated for various types of black holes (see e.g., [25-34] and references therein). It has been disclosed that thermodynamic curvature does not diverge at the critical point, contrary to the fluid systems. Recently, two new normalized thermodynamic curvatures for a charged AdS black hole have been proposed, which diverge at the critical point of the phase transition [35-37]. These thermodynamic curvatures are constructed via the heat capacity at constant volume [35,36] and adiabatic compressibility [37] and have the same behavior for large black holes. In [37] it was shown that the normalized thermodynamic curvature diverges to positive infinity for extremal 
black holes. More recently, the behavior of these two normalized thermodynamic curvatures was studied for several different black holes [38-43].

In this paper, we explore the thermodynamic phase structure of four-dimensional rotating AdS black holes. We consider an extended phase space in the pressure $(P)$ and entropy $(S)$ plane, in which the small-like and largelike black holes are separated by the maximum of the specific heat at constant pressure in the supercritical region. In addition, we provide simple analytical expressions for critical quantities. From the thermodynamic fluctuation metric in the entropy representation, we obtain a Ruppeiner line element of rotating AdS black holes in the pressureentropy coordinates, where it is also valid for the ordinary thermodynamic systems, such as the simple Van der Waals fluid. Then, by using the thermodynamic curvature, we explore the microscopic properties of the system and compare them with those of the Van der Waals fluid system. In particular, we investigate the behavior of the maximum of the specific heat at constant pressure and the minimum of the thermodynamic curvature for these systems in the supercritical region. We find that, for both cases, the thermodynamic curvature diverges at the critical point and goes to positive infinity for the extremal black holes. Finally, the critical behavior of the thermodynamic curvature for the characteristic curves is studied and the critical exponents are calculated.

The rest of the paper is organized as follows. In Sec. II, we first give a brief review on thermodynamics of fourdimensional rotating AdS black holes in the extended phase space and then determine the thermodynamic phase structure in the $P-S$ plane. Next, we obtain the Ruppeiner metric in $(P-S)$ coordinates, and using this, we study in detail the microscopic properties of the black hole and Van der Waals system in Sec. III. Section IV is devoted to investigating the thermodynamic curvature near the critical region. In Sec. V, we present our summary and discussion. In the Appendix, we calculate the thermodynamic curvature of the Van der Waals system using the Ruppeiner metric in $(P-S)$ coordinates.

\section{THERMODYNAMIC PHASE STRUCTURE}

Let us begin with a brief review of the thermodynamics of single spinning AdS black holes in four dimensions, based on Refs. [14,44]. The mass of the Kerr-AdS black hole with pressure $(P)$ is [14]

$M(S, P, J)=\frac{1}{2} \sqrt{\frac{(1+8 S P / 3)\left[4 \pi^{2} J^{2}+S^{2}(1+8 S P / 3)\right]}{\pi S}}$,

where $S$ and $J$ are the entropy and angular momentum, respectively. We also take $(S, J, P)$ as the set of thermodynamic parameters. This assumption can be justified if one extends the phase space by treating the cosmological constant as the thermodynamic variable, pressure, with its conjugate variable as volume [18]. Next, we identify the black hole mass as enthalpy and write down the first law of thermodynamics as

$$
d M=T d S+\Omega d J+V d P,
$$

where $T$ is the Hawking temperature, $\Omega$ the angular velocity, and $V$ the thermodynamic volume, which are given by

$$
\begin{gathered}
\Omega=\frac{\pi J}{\mathrm{SM}}(1+8 S P / 3), \\
V=\frac{2}{3 \pi M}\left(S^{2}[1+8 S P / 3]+2 \pi^{2} J^{2}\right), \\
T=\frac{1}{8 \pi M}\left([1+8 S P / 3](1+8 S P)-4 \pi^{2} J^{2} / S^{2}\right) .
\end{gathered}
$$

The internal energy $U$ is obtained from $M$ via the Legendre transformation, $U=M-P V$, and it is given by

$$
\begin{aligned}
U(S, V, J)= & \left(\frac{\pi}{S}\right)^{3}\left\{\left(\frac{3 V}{4 \pi}\right)\left(\frac{S^{2}}{2 \pi^{2}}+J^{2}\right)\right. \\
& \left.-J^{2} \sqrt{\left(\frac{3 V}{4 \pi}\right)^{2}-\left(\frac{S}{\pi}\right)^{3}}\right\} .
\end{aligned}
$$

In this representation, the first law of black hole thermodynamics is written as

$$
d U=T d S+\Omega d J-P d V .
$$

Note that the above first law is not valid for asymptotically flat black holes, where there is no pressure term in the first law of black hole thermodynamics.

Now, we study the critical behavior of the rotating AdS black hole by investigating the specific heat at constant pressure

$$
C_{P}=\left.T \frac{\partial S}{\partial T}\right|_{P}
$$

where we have also fixed $J$. For constant $J$ and $P=P_{c}$, the value of the critical point can be determined by an inflection point

$$
\left.\frac{\partial T}{\partial S}\right|_{P_{c}}=0,\left.\quad \frac{\partial^{2} T}{\partial S^{2}}\right|_{P_{c}}=0
$$

Using the temperature formula in Eq. (5), the critical quantities are obtained analytically as 


$$
\begin{aligned}
S_{c} & =\frac{24 \pi J}{(73+6 \sqrt{87})^{1 / 3}+(73-6 \sqrt{87})^{1 / 3}-5} \approx 28.719 J, \\
P_{c} & =\frac{\left[(73+6 \sqrt{87})^{1 / 3}+(73-6 \sqrt{87})^{1 / 3}-5\right]^{2}}{768 \pi J} \approx 0.003 / J, \\
T_{c}^{2} & =\frac{(6137+768 \sqrt{87})^{1 / 3}-\frac{239}{(6137+768 \sqrt{87})^{1 / 3}}-7}{384 \pi^{2} J} \approx 0.002 / J .
\end{aligned}
$$

These quantities are the same, numerically, as the ones found in Ref. [45]. Here, we present their analytical expressions for the first time in a compact form. For $P>P_{c}$, the specific heat at constant pressure is positive; i.e., the black hole is thermodynamically stable. However, below $P_{c}$, there exists a certain range of quantities for which the specific heat at constant pressure is negative $\left(C_{P}<0\right)$. This corresponds to a thermodynamic instability of the black hole, which is remedied by the Maxwell equal area construction, $\oint V d P=0$, indicating a first order phase transition between small and large black holes. The region of the first order phase transition, which is obtained from the Maxwell construction, is identified in the $P-S$ plane in Fig. 1. The small and large black hole phases are located at the left and right of the shaded region, respectively. In Fig. 1, the extremal black hole curve (corresponding to zero temperature) is denoted by the gray dashed line, and the critical point is indicated by a black solid circle. The left region of the gray dashed curve is physically excluded because the temperature becomes negative.

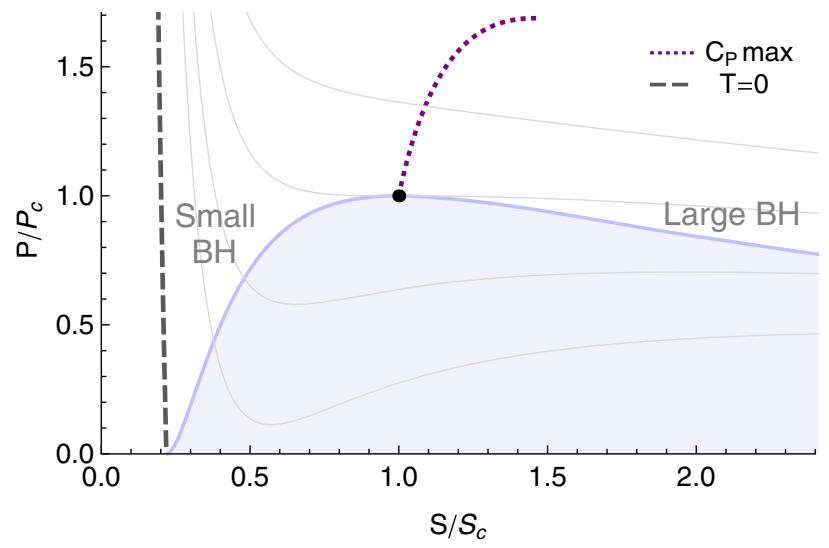

FIG. 1. Phase structure of the rotating AdS black hole in the $P-S$ plane. The coexistence region of the first order phase transition between the large and small black holes is identified. The gray dashed and purple dotted curves correspond to the extremal black hole $(T=0)$ and the local maximum of $C_{P}$, respectively; a black spot represents the critical point. Several isothermal lines are denoted by the thin curves where, from top to bottom, the temperature is decreased. The region on the left of the gray dashed curve is excluded since it leads to negative temperature.
For the supercritical region, which is at higher pressures and entropies than the critical point, we illustrate the local maximum of the specific heat at constant pressure $\left(C_{P}\right)$ in Fig. 1 by the purple dotted line. The local maximum of $C_{P}$ commences from $(\tilde{P}, \tilde{S}) \approx(1.69,1.45)$ and terminates at the critical point, where it goes to infinity, and $\tilde{P}=P / P_{c}$ and $\tilde{S}=S / S_{c}$ are the reduced pressure and entropy, respectively. This curve can be viewed as an extension to the coexistence line, which divides the supercritical region into two phases [46,47]. Here, the small-like and large-like black holes are separated by the local maximum of $C_{P}$ in the supercritical region beyond the critical point.

\section{THERMODYNAMIC CURVATURE}

To set up a thermodynamic Riemannian geometry, we consider the rotating AdS black hole in the canonical (fixed $J$ ) ensemble of extended phase space so that its thermodynamic state is specified by the internal energy $U$ and volume $V$. The line element of the geometry, which characterizes the distance between thermodynamic states, is given by [1]

$$
d l^{2}=-\frac{\partial^{2} S}{\partial x^{\mu} \partial x^{\nu}} d x^{\mu} d x^{\nu}
$$

where $S$ is entropy and $x^{\mu}=(U, V)$. Using the first law for a rotating AdS black hole, Eq. (7), and the Maxwell relation, one can express the line element, Eq. (11), as follows ${ }^{1}$ :

$$
d l^{2}=\frac{1}{T}\left(\frac{\partial T}{\partial S}\right)_{P} d S^{2}-\frac{1}{T}\left(\frac{\partial V}{\partial P}\right)_{S} d P^{2}
$$

By computing the Riemannian curvature scalar $R$ (thermodynamic curvature) from the metric, one can get some information about the interparticle interaction in the thermodynamic system. In particular, the positive (negative) sign of the thermodynamic curvature specifies that the dominant interaction is repulsive (attractive) [2-4]. On the other hand, $R=0$ shows that there is no interaction in the system [49]. In what follows, we examine the behavior of the thermodynamic curvature for the rotating AdS black hole and the Van der Waals fluid.

For the four-dimensional rotating AdS black hole, the thermodynamic curvature is readily calculated as

$$
R=\frac{\mathcal{B}(\tilde{S}, \tilde{P})}{J \tilde{T}\left[(\partial \tilde{T} / \partial \tilde{S})_{\tilde{P}}\right]^{2}},
$$

where $\mathcal{B}(\tilde{S}, \tilde{P})$ is a complicated function of the reduced pressure $(\tilde{P})$ and entropy $(\tilde{S})$ and $\tilde{T}=T / T_{c}$ is the reduced

\footnotetext{
${ }^{1}$ Although this line element is derived for a rotating AdS black hole, it remains valid for an ordinary thermodynamic system [48].
} 


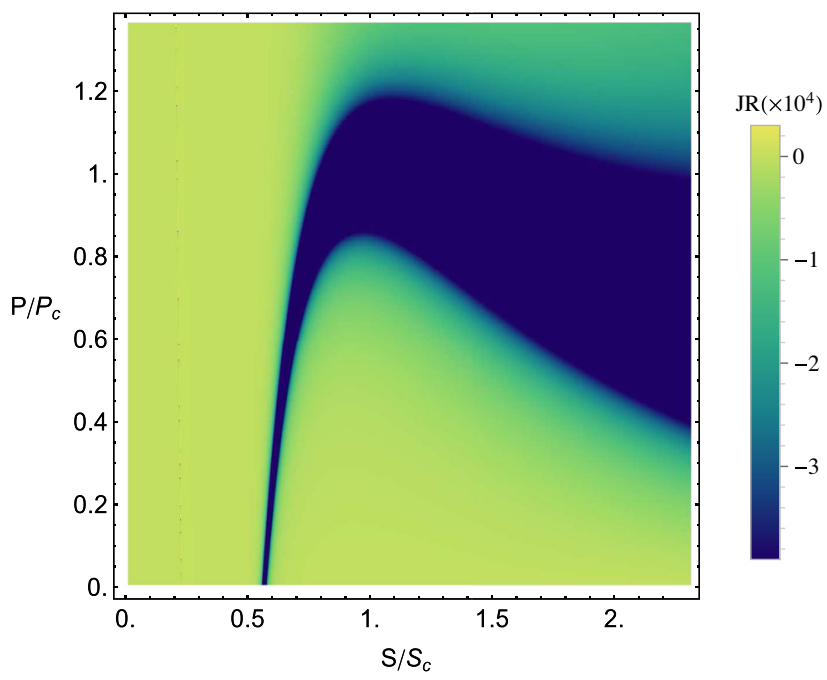

FIG. 2. Behavior of the thermodynamic curvature $R$ for the rotating AdS black hole.

temperature. Note that $R$ is proportional to the inverse of angular momentum in the reduced parameter space. The behavior of $R$ is depicted in Fig. 2 as a function of $P / P_{c}$ and $S / S_{c}$. One can see from Fig. 2 that $R$ is positive in some region of the parameter space. From Eq. (13), $R$ diverges on $\tilde{T}=0$ and $(\partial \tilde{T} / \partial \tilde{S})_{\tilde{P}}=0$, corresponding to the extremal black holes and diverging specific heat at constant pressure, respectively.

In order to examine the thermodynamic curvature more closely, we plot in Fig. 3 the vanishing (brown dotted line) and diverging (gray dashed line) curves of $R$ as well as the

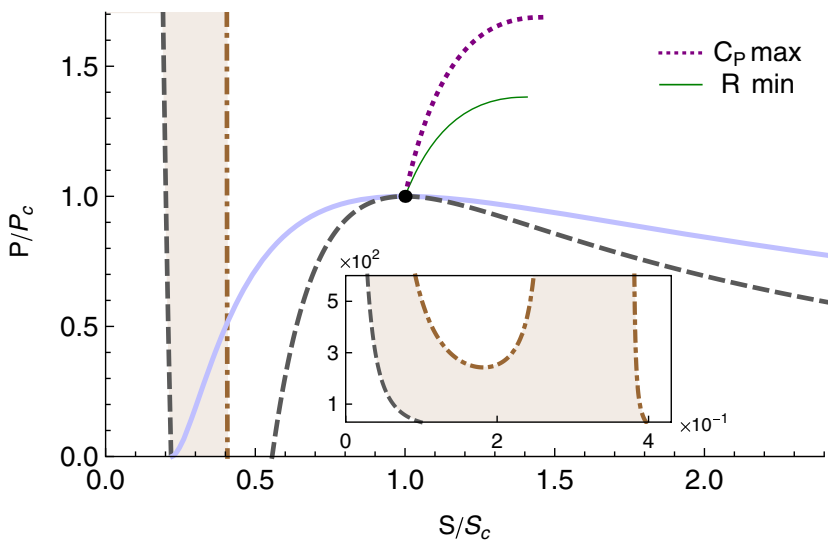

FIG. 3. Coexistence curve (light-blue solid line), diverging curve (gray dashed line), and vanishing curve (brown dotted line) of $R$ for the rotating AdS black hole. The region on the left of the gray dashed curve is excluded since it leads to negative temperature. The thin green and purple dotted curves correspond to the local minimum of $R$ and local maximum of $C_{P}$, respectively; a black spot represents the critical point. In the shaded region, $R$ is positive; otherwise $R<0$. The inset shows a negative $R$ in the shaded region for $\tilde{P} \gtrsim 242.78$. transition curve (light-blue solid line) of small and large black holes and local maximum of $C_{P}$ (purple dotted line), which were already shown in Fig. 1. In Fig. 3, the shaded regions represent positive values of $R$, where the dominant interaction is repulsive. In contrast, $R$ is negative everywhere outside the shaded regions, indicating the dominant attractive interaction. Remarkably, the transition and diverging curves coincide at the critical point, which is highlighted by a black spot. This situation also occurs for ordinary thermodynamic systems [30]. The white area to the left of the gray dashed line on the left side of the figure is excluded because of a negative temperature. One can see from Fig. 3 that the associated $R$ for the large black hole phase is negative. However, for the small black hole phase, there exists a certain region with positive $R$, which is also present in the higher pressure regime. In this region, when approaching the gray dashed curve from above, $R$ diverges to $+\infty$, and the dominant interaction becomes strongly repulsive. The inset in Fig. 3 reveals the existence of a region with negative $R$ in the shaded region when $\tilde{P}$ is greater than $\approx 242.78$. Moreover, in Fig. 3, we also display the local minimum of $R$ in the supercritical region by the thin green line, which begins at $(\tilde{P}, \tilde{S}) \approx(1.41,1.38)$ and ends at the critical point where $R$ goes to negative infinity.

In Fig. 4, we depict the coexistence curve (light-blue solid line) of the Van der Waals vapor-liquid phase transition and maximum of $C_{P}$ (purple dotted line), as well as the diverging (gray dashed line) and minimum (thin green line) of $R$, where the expression of $R$ is given in the Appendix. According to Eq. (A4) and Fig. 4, $R$ has negative values everywhere, indicating the dominant attractive interaction among the molecules. The coexistence and diverging curves coincide at the critical point, which is marked by a black dot. Furthermore, as also seen in Fig. 4, the maximum of $C_{P}$ and minimum of $R$ curves match each

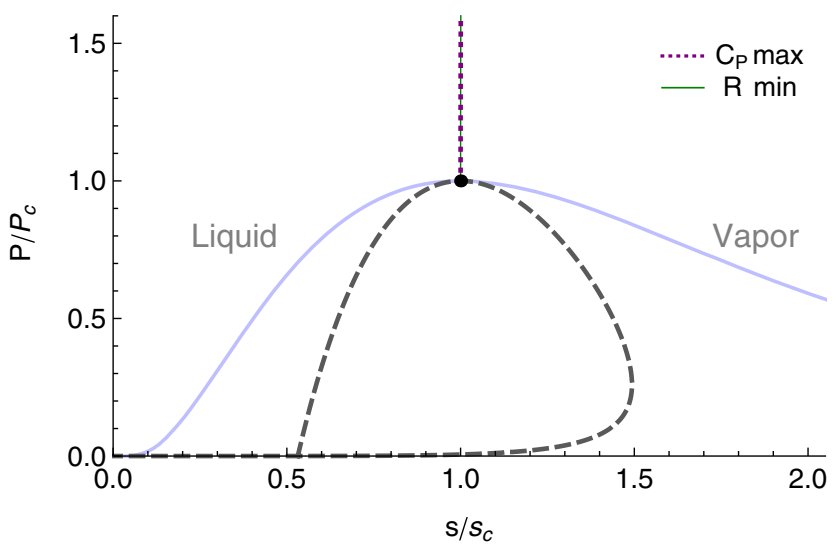

FIG. 4. Coexistence curve (light-blue solid line) and diverging curve (gray dashed line) of $R$ for the Van der Waals fluid. The thin green and purple dotted curves correspond to the minimum of $R$ and maximum of $C_{P}$, respectively; a black spot represents the critical point. The thin green and purple dotted curves match with each other. Note that $R$ is negative everywhere in this plane. 
other in the supercritical region. For the region below the coexistence curve, the Van der Waals model is inapplicable, so it is not considered here.

\section{CRITICAL PROPERTIES}

To further clarify the critical behavior of thermodynamic curvature for the rotating AdS black hole and the associated critical exponent, we investigate the thermodynamic curvature of characteristic curves around the critical point. To do so, in Fig. 5, we illustrate $R$ along its minimum and maximum of $C_{P}$ curves as well as along the transition curve for small and large black holes in the neighborhood of the critical temperature. As is evident from the figure, the large black hole is at higher $|R|$ than the small black hole, and upon approaching the critical point, $R$ in both phases diverges as

$$
R \approx-\frac{41.2}{J}|t|^{-2}
$$

with a universal critical exponent of 2, where $t=T / T_{c}-1$ is the deviation from the critical temperature. In the supercritical regime, the local minimum of $R$ and maximum of $C_{P}$ curves are close together in thermodynamic curvature, and they diverge from above $T_{c}$ as

$$
R \approx-\frac{165.3}{J} t^{-2},
$$

implying a critical exponent of 2 .

For the Van der Waals fluid, the thermodynamic curvature of the vapor and liquid along the coexistence curve near the critical temperature has the following form:

$$
R=-\frac{1}{12}|t|^{-2}
$$

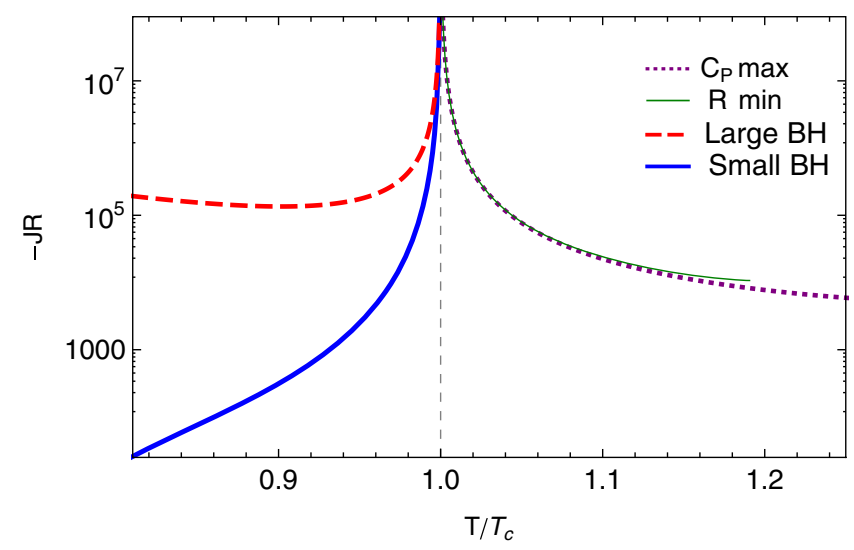

FIG. 5. Thermodynamic curvature $R$ of characteristic curves for the rotating AdS black hole. The purple dotted and thin green curves are close together.
Moreover, upon approaching the critical point from above along the minimum of $R$ and maximum of $C_{P}$ curves, $R$ diverges with the exponent 2 as

$$
R=-\frac{1}{3} t^{-2}
$$

\section{SUMMARY AND DISCUSSION}

Thermodynamic geometry of black holes provides a powerful tool to explore the microscopic structure of these systems and disclose the nature of the interaction between their ingredient particles. In this paper, we have presented simple exact analytical expressions for the critical quantities of the Kerr-AdS black holes and constructed the phase diagram in the pressure-entropy parameter space, where the small black hole and large black hole phases are separated by a first order phase transition region below the critical point. Based on the locus of the maxima of the specific heat at constant pressure, we divided the supercritical region into small-like and large-like black hole regions. Indeed, the line of maxima is used as the Widom line, which is characterized by the maximum of the correlation length. In addition, starting from the Ruppeiner geometry in an entropy representation, we have derived the thermodynamic metric for the Kerr-AdS black holes in the pressureentropy coordinates, which is also valid for any ordinary thermodynamic system. We have explicitly shown that, contrary to the charged AdS black hole [48], the thermodynamic curvature of the Kerr-AdS black hole diverges at the critical point, without needing normalization. Compared to the simple Van der Waals fluid, which has negative thermodynamic curvature everywhere, we have found that there is a certain region for the spinning AdS black holes with small entropy in the space of parameters for which the thermodynamic curvature is positive and the repulsive interaction dominates. Such behavior exists even when the pressure is extremely large. Another distinction is that the dominant interactions in the microstructure of extremal Kerr-AdS black holes are strongly repulsive, which is similar to an ideal gas of fermions at zero temperature [2].

Taking into account the fact that the magnitude of the thermodynamic curvature is related to the correlation length, we have used the locus of the maximum of $|R|$ to characterize the Widom line. We have found that the maximum of $|R|$ is equal to $C_{P}$ maximum values for the Van der Waals fluid in the supercritical region, while for the black hole, they are close to each other near the critical point. Finally, we have determined the critical behavior of thermodynamic curvature of spinning AdS black holes and found out that it is governed by a universal critical exponent of 2, which is the same as the Van der Waals fluid.

In the context of AdS/CFT correspondence, the study of Van der Waals-like phase transitions in AdS black holes 
with a spherical horizon allowed us to investigate the specific aspect on the dual field theories at finite temperature [50,51]. For instance, the hysteretic behavior of the shear viscosity to entropy density of the quantum field theory can be explained by the thermodynamic phase transition of the spherical black holes, where the spherical geometry of the horizon breaks the translational symmetry in the dual quantum field theory. In this paper, the focus was on the phase transition and critical behavior of spinning AdS black holes. The study of hydrodynamic properties of the dual field theory is left for future investigations.

It would be interesting to study reentrant phase transitions and universal properties of higher-dimensional rotating AdS black holes by employing the thermodynamic Riemannian geometry based on the fluctuations of the entropy and pressure.

Note added.-Recently, we learned that another article [52] had addressed the same issue, where it was shown that the thermodynamic curvature has different behavior at small entropy. However, our results differ from [52] in that we find a region within the repulsive interaction area in which the thermodynamic curvature has negative values.

\section{APPENDIX: VAN DER WAALS MODEL}

In this appendix, we calculate the thermodynamic curvature for the Van der Waals fluid in the $P-S$ plane. The specific Helmholtz free energy of the Van der Waals fluid, which contains two parameters $(a, b)$ reflecting the intermolecular interaction and molecular size effects, is given by [53]

$$
F=-\frac{a}{v}-T\left(\ln [v-b]+\frac{3}{2} \ln [T]+\ln [\zeta]+1\right),
$$

where $\zeta=(m / 2 \pi)^{3 / 2}$ and $m$ is the mass of an atom. Here, $T$ and $v$ are the temperature and specific volume, respectively. It is important to note that $v>b$. Using Eq. (A1), the pressure and entropy are obtained as

$$
\tilde{P}=\frac{8 \tilde{T}}{3 \tilde{v}-1}-\frac{3}{\tilde{v}^{2}}, \quad \tilde{s}=\frac{\tilde{T}(3 \tilde{v}-1)^{2 / 3}}{2^{2 / 3}},
$$

expressed in terms of the reduced thermodynamic variables

$$
\tilde{T}=\frac{T}{T_{c}}, \quad \tilde{P}=\frac{P}{P_{c}}, \quad \tilde{v}=\frac{v}{v_{c}}, \quad \tilde{s}=\frac{s}{s_{c}},
$$

where $s \equiv e^{(2 S-5) / 3} / \zeta^{2 / 3}$ and $S$ is the entropy. The critical quantities are

$P_{c}=\frac{a}{27 b^{2}}, \quad v_{c}=3 b, \quad s_{c}=\frac{2^{11 / 3} a}{27 b^{1 / 3}}, \quad T_{c}=\frac{8 a}{27 b}$.

Using the line element in $(P-S)$ coordinates, Eq. (12), the thermodynamic curvature is obtained as

$$
R=\frac{(3 \tilde{v}-1)^{8 / 3}\left[(3 \tilde{v}-1)^{8 / 3}-2^{11 / 3} \tilde{s} \tilde{v}^{3}\right]}{3\left[(3 \tilde{v}-1)^{8 / 3}-2^{8 / 3} \tilde{s} \tilde{v}^{3}\right]^{2}},
$$

which is independent of $a$ and $b$.
[1] G. Ruppeiner, Riemannian geometry in thermodynamic fluctuation theory, Rev. Mod. Phys. 67, 605 (1995); Erratum, Rev. Mod. Phys. 68, 313 (1996).

[2] G. Ruppeiner, Thermodynamic curvature measures interactions, Am. J. Phys. 78, 1170 (2010).

[3] G. Ruppeiner, Thermodynamic curvature from the critical point to the triple point, Phys. Rev. E 86, 021130 (2012).

[4] H. O. May, P. Mausbach, and G. Ruppeiner, Thermodynamic curvature for attractive and repulsive intermolecular forces, Phys. Rev. E 88, 032123 (2013).

[5] J. D. Bekenstein, Black holes and entropy, Phys. Rev. D 7, 2333 (1973); Generalized second law of thermodynamics in black-hole physics, Phys. Rev. D 9, 3292 (1974).

[6] S. W. Hawking, Particle creation by black holes, Commun. Math. Phys. 43, 199 (1975); Black holes and thermodynamics, Phys. Rev. D 13, 191 (1976).

[7] P. C. W. Davies, The thermodynamic theory of black holes, Proc. R. Soc. A 353, 499 (1977).
[8] S. Hawking and D. N. Page, Thermodynamics of black holes in anti-de Sitter space, Commun. Math. Phys. 87, 577 (1983).

[9] A. Chamblin, R. Emparan, C. V. Johnson, and R. C. Myers, Charged AdS black holes and catastrophic holography, Phys. Rev. D 60, 064018 (1999).

[10] A. Chamblin, R. Emparan, C. V. Johnson, and R. C. Myers, Holography, thermodynamics and fluctuations of charged AdS black holes, Phys. Rev. D 60, 104026 (1999).

[11] A. Dehyadegari, A. Sheykhi, and A. Montakhab, Critical behaviour and microscopic structure of charged AdS black holes via an alternative phase space, Phys. Lett. B 768, 235 (2017).

[12] D. Kastor, S. Ray, and J. Traschen, Enthalpy and the mechanics of AdS black holes, Classical Quantum Gravity 26, 195011 (2009).

[13] B. P. Dolan, The cosmological constant and the black hole equation of state, Classical Quantum Gravity 28, 125020 (2011). 
[14] B. P. Dolan, Pressure and volume in the first law of black hole thermodynamics, Classical Quantum Gravity 28, 235017 (2011).

[15] B. P. Dolan, Compressibility of rotating black holes, Phys. Rev. D 84, 127503 (2011).

[16] B. P. Dolan, The compressibility of rotating black holes in D-dimensions, Classical Quantum Gravity 31, 035022 (2014).

[17] B. P. Dolan, Thermodynamic stability of asymptotically anti-de Sitter rotating black holes in higher dimensions, Classical Quantum Gravity 31, 165011 (2014).

[18] D. Kubiznak and R. B. Mann, $P-V$ criticality of charged AdS black holes, J. High Energy Phys. 07 (2012) 033.

[19] S. H. Hendi and M. H. Vahidinia, Extended phase space thermodynamics and $P-V$ criticality of black holes with a nonlinear source, Phys. Rev. D 88, 084045 (2013).

[20] A. Dehyadegari, A. Sheykhi, and A. Montakhab, Novel phase transition in charged dilaton black holes, Phys. Rev. D 96, 084012 (2017).

[21] S. Gunasekaran, R. B. Mann, and D. Kubiznak, Extended phase space thermodynamics for charged and rotating black holes and Born-Infeld vacuum polarization, J. High Energy Phys. 11 (2012) 110.

[22] N. Altamirano, D. Kubiznak, and R. B. Mann, Reentrant phase transitions in rotating anti-de Sitter black holes, Phys. Rev. D 88, 101502 (2013).

[23] S.-W. Wei and Y.-X. Liu, Triple points and phase diagrams in the extended phase space of charged Gauss-Bonnet black holes in AdS space, Phys. Rev. D 90, 044057 (2014).

[24] R. A. Hennigar, R. B. Mann, and E. Tjoa, Superfluid Black Holes, Phys. Rev. Lett. 118, 021301 (2017).

[25] R.-G. Cai and J.-H. Cho, Thermodynamic curvature of the BTZ black hole, Phys. Rev. D 60, 067502 (1999).

[26] J.-Y. Shen, R.-G. Cai, B. Wang, and R.-K. Su, Thermodynamic geometry and critical behavior of black holes, Int. J. Mod. Phys. A 22, 11 (2007).

[27] J.-L. Zhang, R.-G. Cai, and H.-W. Yu, Phase transition and thermodynamical geometry for Schwarzschild AdS black hole in $\mathrm{AdS}_{5} \times S$ spacetime, J. High Energy Phys. 02 (2015) 143.

[28] J.-L. Zhang, R.-G. Cai, and H.-W. Yu, Phase transition and thermodynamical geometry of Reissner-NordstromAdS black holes in extended phase space, Phys. Rev. D 91, 044028 (2015).

[29] G. Ruppeiner, Thermodynamic curvature: Pure fluids to black holes, J. Phys. 410, 012138 (2013).

[30] G. Ruppeiner, Thermodynamic curvature and black holes, Springer Proc. Phys. 153, 179 (2014).

[31] S.-W. Wei and Y.-X. Liu, Insight into the Microscopic Structure of an AdS Black Hole from Thermodynamical Phase Transition, Phys. Rev. Lett. 115, 111302 (2015); Erratum, Phys. Rev. Lett. 116, 169903 (2016).

[32] M. Kord Zangeneh, A. Dehyadegari, and A. Sheykhi, Comment on "Insight into the Microscopic Structure of an AdS Black Hole from a Thermodynamical Phase Transition", arXiv:1602.03711.

[33] M. Kord Zangeneh, A. Dehyadegari, M. R. Mehdizadeh, B. Wang, and A. Sheykhi, Thermodynamics, phase transitions and Ruppeiner geometry for Einstein-dilaton Lifshitz black holes in the presence of Maxwell and Born-Infeld electrodynamics, Eur. Phys. J. C 77, 423 (2017).
[34] M. Kord Zangeneh, A. Dehyadegari, A. Sheykhi, and R. B. Mann, Microscopic origin of black hole reentrant phase transitions, Phys. Rev. D 97, 084054 (2018).

[35] S.-W. Wei, Y.-X. Liu, and R. B. Mann, Repulsive Interactions and Universal Properties of Charged AdS Black Hole Microstructures, Phys. Rev. Lett. 123, 071103 (2019).

[36] S.-W. Wei, Y.-X. Liu, and R. B. Mann, Ruppeiner geometry, phase transitions, and the microstructure of charged AdS black holes, Phys. Rev. D 100, 124033 (2019).

[37] A. Dehyadegari, A. Sheykhi, and S.-W. Wei, Microstructure of charged AdS black hole via $P-V$ criticality, Phys. Rev. D 102, 104013 (2020).

[38] Z.-M. Xu, B. Wu, and W.-L. Yang, Ruppeiner thermodynamic geometry for the Schwarzschild AdS black hole, Phys. Rev. D 101, 024018 (2020).

[39] A. Ghosh and C. Bhamidipati, Thermodynamic geometry for charged Gauss-Bonnet black holes in AdS spacetimes, Phys. Rev. D 101, 046005 (2020).

[40] A. Ghosh and C. Bhamidipati, Thermodynamic geometry and interacting microstructures of BTZ black holes, Phys. Rev. D 101, 106007 (2020).

[41] S. Mahish, A. Ghosh, and C. Bhamidipati, Thermodynamic curvature of the Schwarzschild-AdS black hole and Bose condensation, Phys. Lett. B 811, 135958 (2020).

[42] A. N. Kumara, C. L. A. Rizwan, K. Hegde, M. S. Ali, and K. M. Ajith, Microstructure of five-dimensional neutral GaussBonnet black hole in anti-de Sitter spacetime via $P-V$ criticality, arXiv:2006.13907.

[43] A. N. Kumara, C. L. A. Rizwan, K. Hegde, M. S. Ali, and K. M. Ajith, Ruppeiner geometry, reentrant phase transition and microstructure of Born-Infeld AdS black hole, Phys. Rev. D 103, 044025 (2021).

[44] M. M. Caldarelli, G. Cognola, and D. Klemm, Thermodynamics of Kerr-Newman-AdS black holes and conformal field theories, Classical Quantum Gravity 17, 399 (2000).

[45] S.-W. Wei, P. Cheng, and Y.-X. Liu, Analytical and exact critical phenomena of $d$-dimensional singly spinning KerrAdS black holes, Phys. Rev. D 93, 084015 (2016).

[46] M. Santoro and F. Gorelli, Structural changes in supercritical fluids at high pressures, Phys. Rev. B 77, 212103 (2008).

[47] G. Ruppeiner, A. Sahay, T. Sarkar, and G. Sengupta, Thermodynamic geometry, phase transitions, and the Widom line, Phys. Rev. E 86, 052103 (2012).

[48] A. Dehyadegari, A. Sheykhi, and S.-W. Wei, Microstructure of charged AdS black hole via $P-V$ criticality, Phys. Rev. D 102, 104013 (2020).

[49] G. Ruppeiner, Thermodynamics: A Riemannian geometric model, Phys. Rev. A 20, 1608 (1979).

[50] M. Cadoni, E. Franzin, and M. Tuveri, Van der Waals-like behaviour of charged black holes and hysteresis in the dual QFTs, Phys. Lett. B 768, 393 (2017).

[51] M. Cadoni, E. Franzin, and M. Tuveri, Hysteresis in $\eta / s$ for QFTs dual to spherical black holes, Eur. Phys. J. C 77, 900 (2017).

[52] S.-W. Wei and Y.-X. Liu, A general thermodynamic geometry approach for rotating Kerr anti-de Sitter black holes, Phys. Rev. D 104, 084087 (2021).

[53] L. D. Landau and E. M. Lifzhitz, Statistical Physics (Pergamon, New York, 1977). 